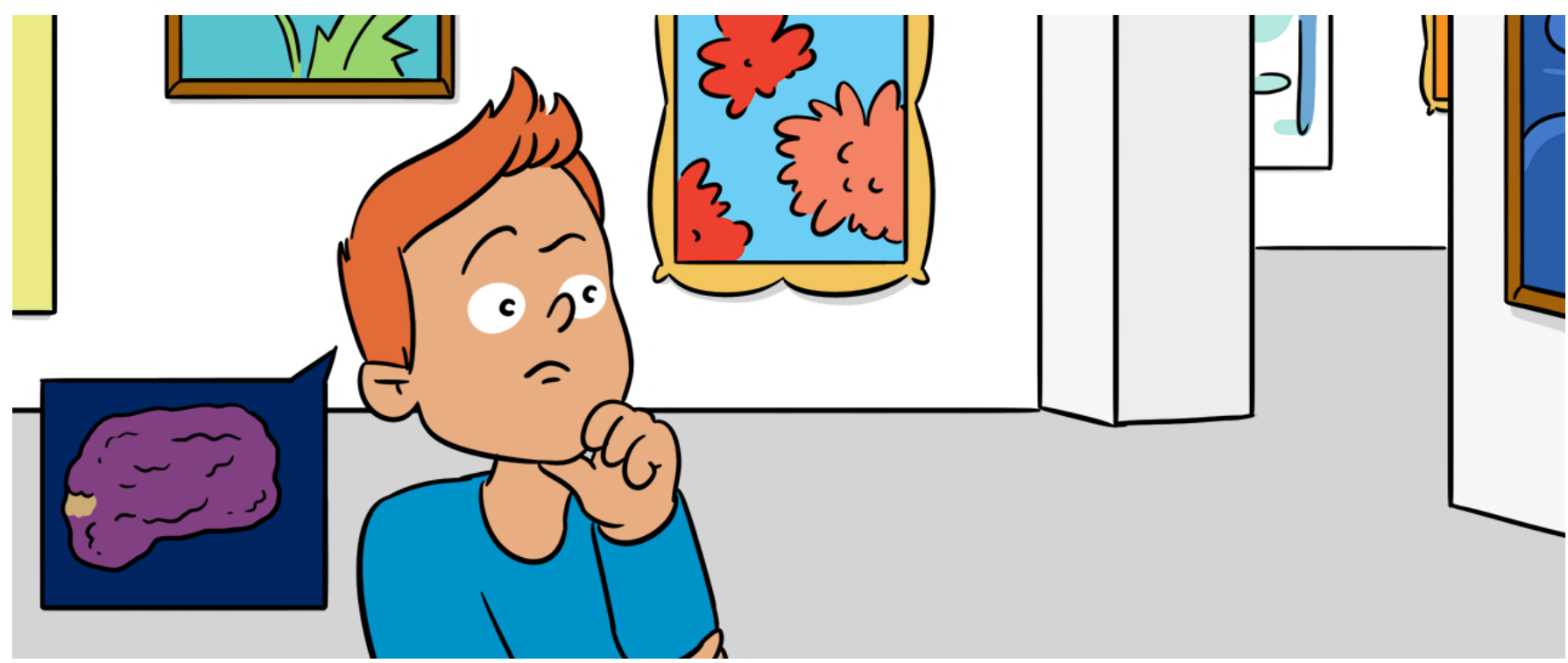

\title{
FAKES AND FORGERIES IN THE BRAIN
}

\section{SCANNER}

\section{Andrew J. Parker*}

Department of Physiology, Anatomy, and Genetics, University of Oxford, Oxford, United Kingdom

REVIEWED BY:

DOUGLAS

AGE: 16

LUAN

AGE: 15

MARIA

EDUARDA

AGE: 15

OLAVO

AGE: 15
When we go to an art gallery, there are usually details about the artworks written on labels on the walls. These labels have been specially prepared by experts to give us their opinions about the art objects. We thought that most people believe what is written on those labels, and we wanted to understand how powerful these opinions could be. So, we put people into a brain scanner to measure their brain responses while they received different opinions about the same pictures. For this research, we used portrait paintings by the famous Dutch artist Rembrandt. Sometimes, we told our viewers that the portrait was a genuine Rembrandt and sometimes we told them the portrait was a fake. When we analyzed the brain responses, the most interesting responses were found when people were told that the paintings were fake. Two parts of the brain, one involved in strategic planning (called the frontopolar cortex) and one involved in vision (called the occipital cortex), seemed to work together when people thought the paintings were fake.

We sometimes hear in the news that a new, precious artwork has been found. This brings a lot of excitement. The new painting, sculpture, or object might be very valuable. It might be significant because no-one knew it existed previously. 
Or it could be significant because the artwork might have disappeared years ago, and everyone thought it was lost forever.

Whether the artwork is a surprise new finding or was simply lost, there needs to be a process of proving that it is the real thing. Nobody likes to be cheated. And nobody likes to be called a cheat. These seem to be basic rules that we learn as we grow up. So, when something is new or unusual, we learn to be careful about claiming that the object is genuine. When the new artwork is finally put on show in a museum or gallery, everybody wants to be sure that it is the real thing.

At the same time, the world around us is full of things we have to take on trust. We trust that cars will stop when the traffic lights are red, because we see that cars actually do stop (almost always). For artworks, it is a little different. Most of us have to rely on what art experts tell us. When we look at the objects on display in a gallery or museum, we assume that they are real. The sign next to the object is placed there to give us a bit more information, and it will usually state what is known about the picture or sculpture. Let us suppose that this sign provides the wrong information. The sign might claim that the artwork is genuine when, in reality, it is a fake. Would that matter?

In one way, it would not matter at all. The visual impression we get from looking at the artwork does not change if the sign next to the artwork is incorrect (Figure 1). Yet, if people find out afterward that they were looking at a fake instead of a genuine work of art, they would feel surprised and perhaps a bit cheated. People viewing the artwork tend to assume that the experts have done their best and are being honest.

This step of deciding whether the artwork is genuine is important. Sometimes, the value of a painting might change by more than 10 times, just based on the words of a couple of experts. We decided that we would try to find out what happens in the brain when people are given different opinions about the same artwork.

\section{FIGURE 1}

This image is made from two half-faces put sideby-side. One half is from a genuine Rembrandt; the other is from a copy. Can you tell which is which? Split image prepared by Martin Kemp.

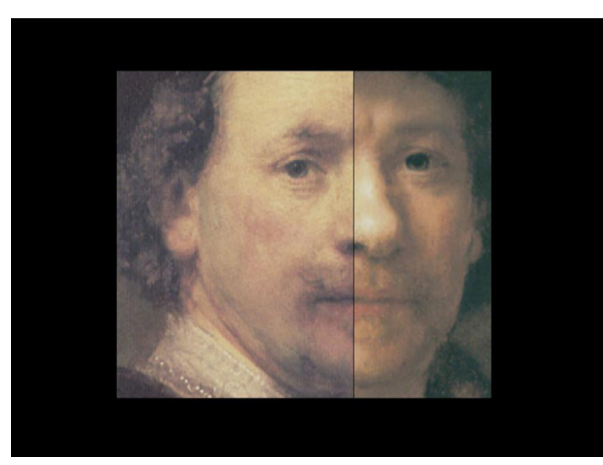

FIGURE 1 
Our team consisted of Mengfei Huang, Holly Bridge, Martin Kemp, and myself. Martin knows about art and Holly and I know about the brain, particularly about brain imaging. But, to be honest, Mengfei did most of the hard work.

Martin advised us to use pictures by the famous Dutch artist, Rembrandt van Rijn. His reason was that there are quite a lot of similar-looking Rembrandt portraits. Some of these are known to be genuine; others are now thought to be painted by someone else. Very few of these other paintings are true forgeries, meaning that someone tried to cheat someone else out of money by making them. Often, they were painted by someone who liked or admired Rembrandt's work and wanted to try something similar for themselves. We called these artworks "copies," not forgeries, because the artists who made them were not deliberately trying to cheat other people.

We were interested in copies that try to copy Rembrandt's style of painting, rather than being a direct copy of a particular painting. These copies have, in past years, deceived a lot of art experts. The Rembrandt project in the Netherlands has only recently begun to sort out all of these tangles about which paintings are genuine and which are not. Art experts call paintings "fake," when they are not genuine.

Mengfei got us a set of images from the Rembrandt project. Some of these images showed genuine Rembrandt portraits but some of them showed fakes. We decided to show both the genuine and fake portraits to people in our brain scanner and then measure their brain activity (see Box 1). Before we did that, we rearranged the labels. We called some of the genuine works "authentic," meaning that they were really painted by Rembrandt, but we called other genuine works "copies," meaning that we incorrectly told people that they were not genuine Rembrandt paintings. For the images from the Rembrandt project that were fakes, we did the same swapping of labels.

So, we ended up with four types of image presentation in the scanner. Here is a table to show this.

By mixing up labels and images in the way shown here, we were able to find out specifically what effect swapping the labels has on brain activity, regardless of whether the image was authentic or a copy.

We then put our participants into the brain scanner (see Box 1) and got them to look at the sequence of images. Just before they saw an image, they heard an audio recording telling them whether the image was "authentic" or a "copy." While they looked at the image, the scanner collected their brain responses. We divided the participants into two groups. Both groups saw all the images. The first group heard a label for each image, according to the mixing up of labels 


\section{BOX 1}

How does the brain scanner work?

The person slides on a narrow bed into the middle of a large tube, which contains a very strong magnet about 10,000 times stronger than the earth's magnetic field. By itself, the earth's magnetic field can pull a compass needle in alignment with North to South. Some atoms have an internal structure that makes them behave like tiny, magnetic compass needles. The brain scanner's main magnet is strong enough to align these tiny atomic magnets. Almost every chemical in the brain has this magnetic structure, but by far, the most common is water.

Placing the person's head in the brain scanner aligns the tiny atomic compass needles in the brain's water with the main magnetic field. The scanner then uses a burst of radio waves strong enough to push a few of these atomic magnets out of alignment. The atoms themselves do not move, but their internal compass needle is altered. When the burst is over, the atomic spin directions fall back to into alignment. The main atoms involved are the hydrogen atoms in the water molecules in the brain. The scanner has a super-sensitive detector to measure these tiny changes in magnetic alignment.

When nerve cells in the brain become active, the body starts sending an extra amount of blood flow to the active parts of the brain. It is almost as if the brain cleverly adjusts the blood flow upwards in anticipation of needing to use more energy. The exact details of how this works are not known yet, but what we do know is that the active regions of the brain have blood that contains more oxygen. Oxygen is carried in the blood by a protein (called hemoglobin) that has some iron in it. In the lungs, the oxygen attaches itself to the iron in the hemoglobin and gets released wherever the body needs oxygen.

Iron is always magnetic, but the iron in the blood becomes more or less magnetic depending on whether oxygen is attached. If the blood is more magnetic, it hides the signal we expect to get from those hydrogen atoms, as we described above.

Therefore, we can figure out that, if the signal from the hydrogen atoms is weaker, then this brain region has more blood with oxygen attached. This shows us which regions of the brain are active at any one moment. The scanner covers the whole brain at once in every single scan, so we can see the whole picture of brain activation using this method. More details about how these pictures are created are in Box 2.

This method only tells us about the average of what a lot of brain cells are doing. If we want to find out more detail about individual brain cells, then we have to use other methods.

and images in Table 1. The second group saw the same images but in every case the label they heard was the opposite of what the first group heard. We tried to keep everything as balanced as we could, so that we were not biasing people one way or the other.

Mengfei then analyzed the brain images (see Box 2), with Holly's help, using the categories in Table 1. In Figure 3, you can see the regions of 


\section{BOX 2}

How do we get an image out of the brain scanner?

\section{FIGURE 2}

How the brain scanner makes an image. The helper magnets change the strength of the magnetic field so that the field is slightly stronger on one side of the person's head. This tunes the structures within the hydrogen atoms of water molecules to ring with different tones. Water molecules in different positions (represented by the bells) send out a slightly different tone. Low tones (large bells) to the right and high tones (small bells) to the left. The scanner uses a pulse of radio waves to set the bells ringing. The scanner's instruments and computer measure the tones coming back from the hydrogen atoms and build a spatial map based on the high and low tones coming back.
The scanner has a couple of helper magnets as well as the main large magnet. These helper magnets vary the strength of the magnetic field at different positions in the scanner. Why is this useful? It turns out that when the internal compass needles of hydrogen atoms fall back into alignment with the main magnetic field, they wobble around. The rate at which this wobbling takes place depends very precisely on the strength of the magnetic field (see Figure 2).

This is where the helper magnets come in. They vary the strength of the magnetic field at each position inside the scanner. The result is that we know that a signal is coming from the water molecules on the left side of the brain because these hydrogen atoms are wobbling at a slightly different rate than the hydrogen atoms on the right side of the brain. This means that each position in the brain gets "tagged" with a different rate of wobble. The scanner can also create images of specific slices of the brain.

The rest of the processing is done by a computer that takes all these different signals, wobbling at slightly different frequencies, and turns them into a meaningful image that we can look at. The computer programme is quite complex, but it is rather like listening to someone playing the piano with your eyes closed. You know that the low bass notes must come from the left-hand end of the piano keyboard and the high notes from the right-hand end, because the piano is arranged that way. The scanner uses its helper magnets to achieve this arrangement.

For more details on how MRI scanners work, see https://www.win.ox.ac.uk/for-thepublic/what-is-fmri.

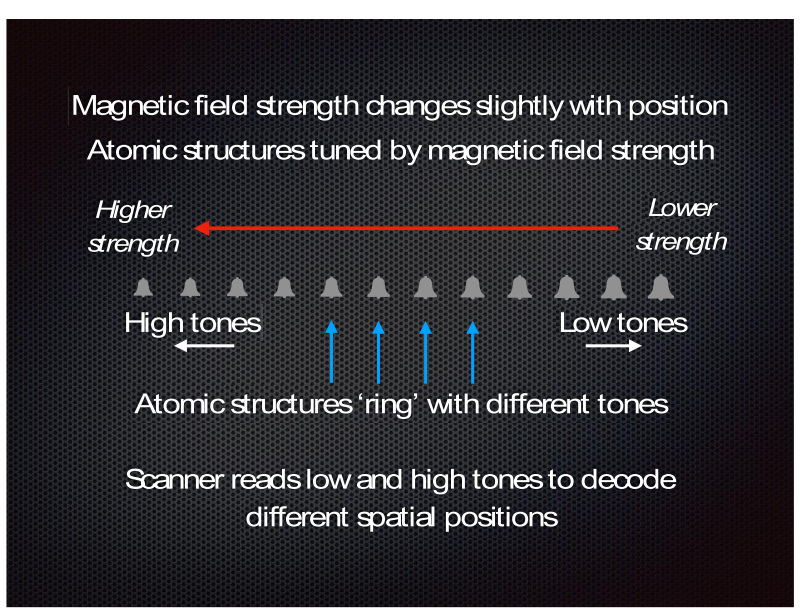

FIGURE 2

the brain that showed up as important in our study. Taking the average across our 14 participants, we found three interesting things. First, when people were told that an image was "authentic" (even when this opinion was not true), a part of the brain called the orbitofrontal cortex became active. Brain scientists already know that this part of the brain responds 
TABLE 1

Different artworks and their labels.

\section{FIGURE 3}

Side view of human brain showing the left side of the brain, with the front of the brain on the left side of the image and the back of the brain on the right. The three brain areas important in this study are marked. The colored area in the occipital cortex showed increased communication with the front of the brain when people were told that they were viewing fake artworks.

\begin{tabular}{ll}
\hline Authentic image labeled "Authentic" & Authentic image labeled "Copy" \\
\hline Copy image labeled "Authentic" & Copy image labeled "Copy" \\
\hline &
\end{tabular}

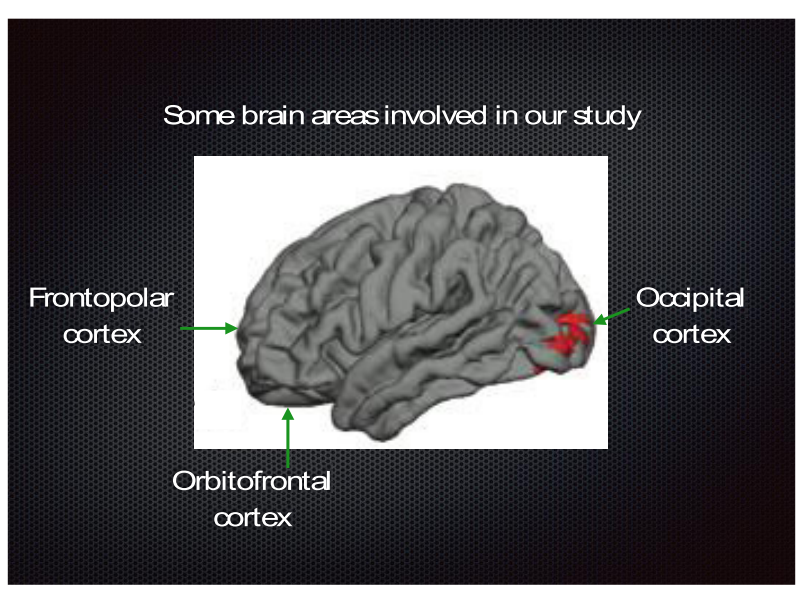

FIGURE 3

when we feel that something is rewarding or pleasurable. This might be anything from eating ice cream to winning at a game of cards. Being told that we are looking at a genuine Rembrandt also seems to be rewarding, based on our results.

Second, we tested whether the brain could tell the difference between a genuine Rembrandt portrait and a copy, regardless of the label. This meant comparing the brain responses from the first row of Table 1 against the brain responses from the second row. There was no meaningful difference. Although this result was a bit of a disappointment, it is still interesting. This result told us that the visual differences between the genuine Rembrandts and the copy Rembrandts were so small that on average people's brains responded in the same way to genuine and fake Rembrandts.

Third, and most interesting of all, were the brain responses to the "copy" label. After having their brains scanned, several people commented that each time they heard a "copy" label being given, they became quite busy trying to work out what made the image a fake, instead of genuine. Their brain responses showed this activity. A part of the brain right at the very front, just behind the forehead bone, became active. This brain area is called the frontopolar cortex. The frontopolar cortex is involved in problem-solving and working out long-term strategies, things that we usually associate with a high degree of intelligence. Brain scientists do not know everything that goes on in the frontopolar cortex, so it is interesting that it became active in our experimental situation. 
We also found out another thing about the activity of the frontopolar cortex. Its activity became linked to the activity of the visual parts of the brain. The visual parts of the brain are at the opposite end of the brain from the frontopolar cortex; they are at the back of the head, in a region called the occipital cortex. What we discovered was that, if the activity of the occipital cortex went up or down, then the activity of the frontopolar cortex went up or down at the same time. But this only happened reliably when the participants were told that the painting was a copy. It is as if these two parts of the brain started talking to each other more strongly when people were given the copy label.

According to our experiments, making a judgment about whether or not something is genuine involves several different parts of the brain working together, rather than just one part of the brain becoming active all on its own. We like this idea and feel that it is the next thing to test more rigorously. We also have to remember that our participants were not looking at real artworks during this study. They were only looking at images of the artworks on a computer screen. This could be a limitation of what we did, but all scientific studies involve some degree of compromise.

We also had a lot of fun doing this research and starting to work out these new ideas. If you feel ready to look at the actual scientific paper that we wrote about this, you can look here [1]. What about our original question? Does it matter if we are cheated by an artwork? The answer seems to be, "Yes, it does," and it is a bit more complicated than just where the paint is placed on the artwork. What we have shown is that what people think about artworks is very important. When we give different opinions about the same artwork, people seem to change their own opinions and their attitude. By scanning their brains, we were able to find out something about what people are doing when their opinion is changed by new information.

\section{ORIGINAL SOURCE ARTICLE}

Huang, M., Bridge, H., Kemp, M. J., and Parker, A. J. 2011. Human cortical activity evoked by the assignment of authenticity when viewing works of art. Front. Hum. Neurosci. 5:134. doi:10.3389/fnhum.2011.00134.

\section{REFERENCE}

1. Huang, M., Bridge, H., Kemp, M. J., and Parker, A. J. 2011. Human cortical activity evoked by the assignment of authenticity when viewing works of art. Front. Hum.

Neurosci. 5:134. doi:10.3389/fnhum.2011.00134 
SUBMITTED: 20 August 2016; ACCEPTED: 26 July 2018;

PUBLISHED ONLINE: 17 August 2018.

EDITED BY: Sabine Kastner, Princeton University, United States

CITATION: Parker AJ (2018) Fakes and Forgeries in the Brain Scanner. Front. Young Minds 6:39. doi:10.3389/frym.2018.00039

CONFLICT OF INTEREST STATEMENT: The author declares that the research was conducted in the absence of any commercial or financial relationships that could be construed as a potential conflict of interest.

COPYRIGHT (C) 2018 Parker. This is an open-access article distributed under the terms of the Creative Commons Attribution License (CC BY). The use, distribution or reproduction in other forums is permitted, provided the original author(s) and the copyright owner(s) are credited and that the original publication in this journal is cited, in accordance with accepted academic practice. No use, distribution or reproduction is permitted which does not comply with these terms.

\section{REVIEWED BY}

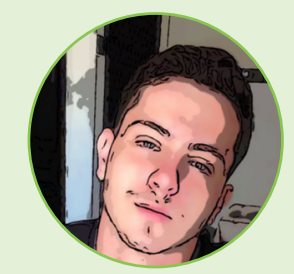

\section{DOUGLAS, AGE: 16}

Hi!, I am in the 12th grade at "Coronel Arthur Pires" State High School. My favorite classes are biology, history, and languages. I have been playing volleyball since I was 9 years old. I want to become a physician and participate in the Doctors Without Borders initiative.

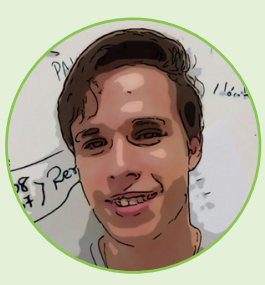

\section{LUAN, AGE: 15}

Hi! I am in the 10th grade at Coronel Arthur Pires' State High School, a public state secondary school. My favorite subjects in school are biology and chemistry. I like to play basketball, play guitar, hangout with friends, and participate in church activities.

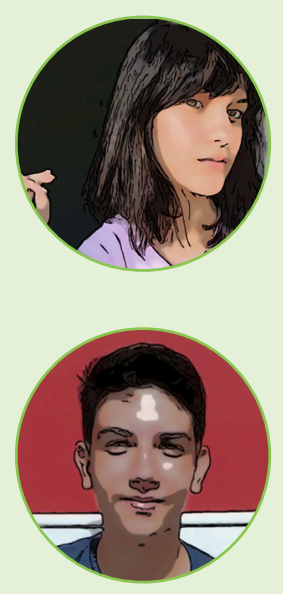

\section{MARIA EDUARDA, AGE: 15}

Hil I am in the 10th grade at Coronel Arthur Pires' State High School. My favorite subjects in school are biology and philosophy. In my free time, I like dancing ballet, reading, and rollerblading.

\section{OLAVO, AGE: 15}

I am in the 10th grade at Miguel Nayme High School located at São Simão city. Math and Physics are my favorite subjects. After school I enjoy reading, playing guitar, and video games. After completing secondary education, I want to study aeronautical engineering. 


\section{AUTHOR}

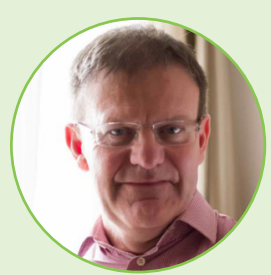

\section{ANDREW PARKER}

Andrew Parker studied Natural Sciences at Cambridge England and stayed to do a Ph.D. When he moved to Oxford in 1979, his father kept asking whether all this research was "actually leading anywhere." These questions kind of stopped about 17 years later when he was made a Professor. Andrew's main interest is the special sensation of stereo depth you get when looking at 3-D movies in the cinema. His main tip in science is "never trust anyone who thinks they have a grand theory of everything.” *andrew.parker@dpag.ox.ac.uk 